\title{
Strates
}

STRATES Matériaux pour la recherche en sciences sociales

5 | 1990

Conjuguer stratégies et territoires?

\section{Stratégies territoriales : valeurs et usages dans les recherches rurales et agroalimentaires de STRATES}

\section{Françoise Plet}

\section{(2) OpenEdition \\ 1 Journals}

Édition électronique

URL : http://journals.openedition.org/strates/1406

ISSN : $1777-5442$

\section{Éditeur}

Laboratoire Ladyss

\section{Édition imprimée}

Date de publication : 31 décembre 1990

ISSN : 0768-8067

\section{Référence électronique}

Françoise Plet, «Stratégies territoriales : valeurs et usages dans les recherches rurales et

agroalimentaires de STRATES », Strates [En ligne], 5 | 1990, mis en ligne le 16 mars 2007, consulté le 07 septembre 2020. URL : http://journals.openedition.org/strates/1406

Ce document a été généré automatiquement le 7 septembre 2020.

Tous droits réservés 


\title{
Stratégies territoriales : valeurs et usages dans les recherches rurales et agroalimentaires de STRATES
}

\author{
Françoise Plet
}

\section{Stratégie et politique}

1 Beaucoup de choses ont été dites sur la question de stratégie et sur la relation entre stratégie et politique. Cette relation paraît immédiate, car une définition courante de stratégie est conduite et réalisation par les meilleurs moyens d'une politique. Cette relation directe ne paraît pas dans un premier temps devoir être beaucoup discutée. Jeanne Fagnani a adopté des définitions de type a-militaire ${ }^{1}$ que je ne reprendrai pas tout à fait : je voudrais simplement dire que quand elle définit stratégie uniquement par des réactions à des conflits, cela apparaît comme une conception réductrice, liée à la guerre du XVIII siècle et aux premières définitions qui n'ont plus cours aujourd'hui ; quand elle parle de déroulement sur un long terme, il est certain que stratégie a une connotation dischronique, une dimension projective, mais le terme n'est pas forcément long; il faudrait préciser le mot.

2 Une stratégie peut être positive (non pas au sens moral, mais au sens de la réalisation d'une action, d'un changement), ou défensive, conservatrice, pour que tout reste en l'état. L'idée de coordination d'acteurs ou de conduites parcellaires n'en est pas absente. Quand on parle de stratégie, il ne s'agit pas nécessairement d'impulsions extérieures. Il peut s'agit de la gestion d'évolutions spontanées, de leur coordination, de l'assignation d'un but à des comportements ou à des initiatives, et la notion apparaît beaucoup plus étendue que la définition restrictive de l'art militaire du $\mathrm{XVIII}^{\mathrm{e}}$ siècle. Elle n'en apparaît pas moins privilégier l'impulsion par rapport au fonctionnement.

Stratégie territoriale

Quant à stratégie territoriale, le groupe de mots nous parait limiter considérablement le champ d'emploi de la notion de stratégie, notamment si l'on accepte une définition un peu restrictive de territoire comme objet spatial socialement construit et délimité. Comme beaucoup d'outils importés, cette notion est délicate à utiliser et dans la 
pratique on constate qu'il s'agit souvent, dans des textes de géographes ou d'autres, de groupe de mots à tout faire, d'une affirmation un peu a priori qui évite de poser des problèmes ou d'aller un peu plus loin dans le décryptage de phénomènes. D'ailleurs, spatial et territorial sont souvent utilisés comme synonymes, pour des raisons stylistiques plus que scientifiques. Ou bien l'on admet que les termes de territoire, territorial, sont chargés d'une forte dimension symbolique assise sur des notions de domaine, de souveraineté, de possessions de biens, et donc leur utilisation est souvent idéologique ; ou bien ils tendent à remplacer dans le langage courant, et notamment associatif, les mots local ou pays qui apparaissent un peu vieillis. À ce moment-là, on accepte un sens très large qui correspond à la dynamique naturelle du langage.

4 Pour nombre de phénomènes spatiaux étudiés, on est souvent dans l'incapacité d'utiliser la notion de stratégie territoriale, soit parce que ne s'en dégage pas l'impression claire de stratégies, soit parce que les stratégies observées - ou repérées - n'ont pas de dimension territoriale, au sens de projet sur la transformation - ou le maintien en l'état - d'un espace construit ou pensé comme territoire (au sens de construit vécu). La combinaison est très restrictive car toute stratégie ne vise pas un territoire, ni n'affecte systématiquement un territoire ; elle peut être spatiale et non territoriale, ce qui est déjà plus large, ou affecter indirectement l'espace. Il y a des stratégies de localisation, qui ont une dimension spatiale sans être territoriale. Il importe donc, dans l'identification des stratégies d'acteurs, de vérifier la nature de ces stratégies et de les examiner de manière précise avant de décider si elles sont spatiales, territoriales, à effet sur des territoires ou du moins des espaces, ce qui est le cas le plus fréquent, car il paraît extrêmement rare qu'une action n'affecte pas cette dimension commune des sociétés que représente la spatialité.

$5 \quad$ Il est peut-être possible de se contenter d'action territoriale, plus large, terme employé par Philippe Pinchemel, qui n'utilise jamais le mot stratégie (La Face de la Terre $)^{2}$. Une collectivité territoriale a une action territoriale, mais pas dans tous les cas une stratégie, et le plus souvent il est difficile de penser en ces termes.

6 Les termes de stratégie et système ont été évoqués par Yvan Chauviré à propos de scénario d'avenir ou du moins de devenir. Le fonctionnement spatial peut être une résultante de mouvements divers, de coïncidences qui construisent des territoires que l'on peut considérer comme des combinaisons dynamiques, un peu au hasard, sans plan ni tension, vers la réalisation d'un plan; dans ce cadre-là, on opposerait volontiers stratégie spatiale ou territoriale à analyse en termes de systèmes qui privilégierait le fonctionnement par rapport à des actions vers des changements ou des conservations. Cela n'est pas simple, car à moins de connaître des stratégies a priori, d'en être conscient, de les avoir bien définies ou qu'elles aient été définies par les acteurs, il est parfois difficile de déterminer, dans ce qu'on observe sur les territoires, s'il y a ou non stratégie. Des stratégies conservatoires notamment peuvent ne pas du tout être manifestes, cachées qu'elles nous sont par le fait que les territoires étudiés nous paraissent stables et fonctionner de manière tout à fait normale ${ }^{3}$. La complexité fait partie du terme et se retrouve dans la définition de la notion de stratégie, car elle doit s'appliquer à éliminer les conséquences jugées négatives d'un objectif et elle incorpore des variables dont les plus importantes ne sont pas forcément rationnelles ni quantifiables.

7 Jeanne Fagnani a également fait allusion à la théorie des jeux; je n'y reviendrai que pour dire que la théorie des jeux montre, entre autres, le rôle des noyaux de coalition, 
d'interaction et de lien au sein de ceux-ci dans des espoirs de gain, maintien ou rupture d'équilibre - c'est-à-dire la stratégie en œuvre. La description des stratégies territoriales en œuvre à Brasilia faite par Michel Rochefort ${ }^{4}$ le montre. On peut rappeler le jeu classique des poubelles : le but du jeu, pour un ensemble de joueurs qui possèdent chacun un jardin propre et une poubelle pleine, consiste à essayer de se débarrasser de sa poubelle en gardant son jardin propre ; la stratégie la plus évidente est de constituer une coalition qui aboutisse à mettre toutes les poubelles dans le jardin d'un seul, isolé et marginal. Tout le monde aura reconnu la pratique territoriale commune de localisation des déchets : les usines d'incinération des ordures ménagères sont localisées à la périphérie des villes et dans des quartiers pauvres en urbanisation, sauf à Rouen où visiblement personne n'a réfléchi une minute avant d'utiliser un espace vacant ; les régions recherchent à leur périphérie, pour les déchets industriels, des espaces ruraux vieillis qui présentent une faible capacité d'opposition autonome (fatalisme, information faible), et qui, par leur position géographique (la périphérie), minimisent les nuisances pour l'espace de souveraineté, la région, l'État... L'exportation de produits dangereux vers le Tiers Monde procède de la même logique. En amont du " jeu », de la "stratégie de gain » utilisée, existe un modèle de représentation des espaces ruraux, des espaces pauvres comme interstitiels, utilitaires, et non pas comme des territoires socialement construits, à considérer, à ménager... Dans les cas complexes, analyser ou chercher à reconnaître des stratégies territoriales ou spatiales ne peut se passer de l'identification des éléments de l'ensemble considéré, de la nature et de la force des interactions qui les relient. Évaluer des stratégies identifiées ou a priori connues et notamment leur rôle dans les dynamiques des espaces ne peut également se passer d'une connaissance approfondie de leurs espaces d'application.

D'ailleurs l'intitulé complet du laboratoire STRATES ne fait-il pas explicitement référence à ce projet ambitieux de comprendre les transformations des espaces géographiques, non pas en termes d'analyse rétrospective traditionnelle, mais en termes d'évaluation des forces de changement internes et externes, de leur plus ou moins grande sensibilité ou "fragilité " (mot à la mode lui-même mal défini) à des modifications des interactions en jeu, modifications qui peuvent être le fait de stratégies qui leur sont appliquées, bref à tendre vers la prédiction de situations nouvelles potentielles?

Les travaux de l'équipe « Systèmes de pouvoirs et dynamiques des espaces ruraux »

Nos pratiques et nos résultats actuels au sein de l'équipe sont plus modestes pour des raisons qui apparaissent surtout tenir aux nécessités qui nous sont faites ou que nous nous imposons en termes de travail multidisciplinaire, de participation à des réseaux, de contrat et de production de rapports. Ces nécessités pratiques réduisent le temps d'approfondissement de l'exploration de nos champs de recherche, les résultats que nous donnons restant peut-être un peu trop des premières analyses.

* Un premier constat est que l'entrée en termes de stratégie territoriale ou spatiale n'est pas dominante parmi nos recherches. Par contre, paradoxalement, l'analyse des politiques (comparaison des politiques d'aménagement rural Espagne/France, Pologne/ France; développement local) y figurent en bonne place. Pourquoi alors la notion de stratégie ne s'impose-t-elle pas? L'application de ces politiques est-elle trop diluée, les politiques elles-mêmes trop peu convaincues ou convaincantes ? Et si oui, cette absence de conviction n'est-elle pas stratégique ? Ceci invite à aller plus loin. 
11 * L'aspect observatoire, par l'intermédiaire du P.I.R.E.N., puis de l'axe AGRAL/STRATES : "Évolution des exploitations agricoles dans leur contexte spatial du local au régional ", privilégie finalement une analyse des comportements et de réactions d'acteurs dans des contextes en changement, ces comportements n'étant pas considérés comme des stratégies. On cherche plutôt à comprendre des fonctionnements socio-spatiaux dans le cadre de petites régions généralement très rurales et qui ne figurent pas parmi les principaux lieux d'émergence des innovations agricoles actuelles. On met à jour des mécanismes d'évolution territoriale au sein desquels les politiques figurent parmi les éléments et dans les interactions, mais sans que, semble-t-il, on n'y lise de stratégie.

12 Pourtant, en Boischaut, Yves Luginbuhl ${ }^{5}$ observe que certains groupes d'agriculteurs mettent en œuvre des stratégies foncières de regroupement des terres.

13 Marie-Claude Guerrini ${ }^{6}$ s'est jusque-là surtout préoccupée de l'identification des acteurs en Aigoual, mais présuppose l'existence de stratégies passées ou en œuvre, encore à reconnaître et à étudier. Nicole Mathieu n'observe pas de stratégie territoriale sur le Méjan, mais pense que dans le fonctionnement d'espaces locaux, il peut y avoir des stades au cours desquels des stratégies territoriales peuvent jouer un grand rôle, et d'autres de calmes stratégiques, liés à l'état des sociétés et de leurs enjeux. La notion de systèmes de pouvoirs, avancée dès le rapport DISPE $(1983)^{7}$ pour comprendre à ce niveau les jeux des acteurs, apparaît cependant plus opératoire à ceux d'entre nous qui centrent particulièrement leur effort sur l'étude du local.

14 * Les entrées par la perception de la nature et des paysages, le travail et l'emploi, les comportements politiques dans les milieux ruraux ne s'examinent évidemment pas immédiatement en termes de stratégies, d'une part parce que la phase descriptive préalable y est extrêmement importante, d'autre part parce que ces travaux, contrats ou préoccupations personnelles, n'ont pas été entrepris dans cette perspective, même s'ils mettent à jour des dynamiques spatiales.

15 * En revanche, il est des entrées évidentes de longue date à quoi la nature même des objets de recherche invite immédiatement. Il s'agit de stratégies foncières et de stratégies d'entreprises.

16 - Le terme stratégies foncières est depuis longtemps utilisé pour nommer les objectifs et les rapports de forces en œuvre pour la disposition des terres et notamment des terres agricoles. Il est surtout employé à propos d'expansion de surfaces ou de concentration spatiale par des groupes sociaux ou des entités familiales (regroupements parcellaires actuels du Boischaut, initiés et mis en œuvre par des groupes bien définis; expansion dans le Bassin Parisien, puis dans quelques régions choisies du reste de la France, enfin à l'étranger, des héritiers des grandes familles agricoles constituées parfois dès le $\mathrm{XVIII}^{\mathrm{e}}$ siècle...). Il s'agit bien ici de stratégies d'agrandissement de l'aire d'activité, ou de rationalisation de cette activité, de stratégies spatiales de manière certaine, territoriales si l'on admet que les entités «famille» ou "exploitation agricole» représentent des pouvoirs organisateurs qui permettent de parler de territoire.

17 - Le second domaine évident est celui des dynamiques agroalimentaires. Il n'y a aucune ambiguïté si l'on considère le fonctionnement des groupes aujourd'hui de par le monde. Ils recherchent des aires de marché définies notamment par des marques et des natures de produits qui varient assez sensiblement dans l'espace en fonction des habitudes alimentaires. Les stratégies de croissance externe - achats d'entreprises, accords 
financiers et techniques - servent ces objectifs d'expansion spatiale de l'activité. Depuis 1984-85, il est possible par exemple d'affirmer que la stratégie de BSN est une stratégie européenne visant pour l'ensemble des produits élaborés, des yaourts aux nouilles, à disposer d'unités de production et de marques dans l'ensemble de l'espace de libreéchange. C'est d'abord la constitution d'un centre, Italie, Espagne, RFA, en relation de voisinage immédiat avec la France, qui a été visé, puis plus tard, la zone GrandeBretagne/Pays-Bas, et la Grèce. Il est légitime de considérer que cette stratégie est spatiale vue de l'extérieur, mais bien territoriale du point de vue de l'entreprise qui vise à se constituer un territoire cohérent au plan des localisations, comme à celui de l'équilibre de la répartition de ses diverses activités. Si l'on observe plus généralement les stratégies en Europe, il est possible de reconnaître des sous-ensembles régionaux particuliers relevant d'aires culturelles et de relations commerciales anciennes, qui guident des stratégies particulières. Les groupes australiens visent plus volontiers la zone anglosaxonne, les groupes britanniques, les Pays-Bas et le Portugal, les groupes allemands, généralement plus petits, la Grèce et l'Italie... ou les Néerlandais l'Indonésie...

Les entreprises de champagne, orientées, à travers les groupes auxquels elles appartiennent, vers la consommation de luxe dans le monde entier, mettent en œuvre, elles, une stratégie spatiale différente, visant à l'expansion de leur offre en vins, légalement limitée (et dont pratiquement tout le potentiel est actuellement atteint) en appellation "Champagne». Elles implantent à cet effet domaines et unités de production dans les grands vignobles du monde pour élaborer surtout des mousseux à leur marque : en Argentine, dans la Napa Valley, la Rioja en Espagne, en Australie et Nouvelle-Zélande.

On vient d'évoquer des stratégies à la fois concurrentes et semblables. Si l'on observe le secteur de l'industrie de la viande en France, il apparaît nettement une opposition entre les stratégies des collectivités locales et celles des groupes. Pour les premières, il s'agit de conforter, voire de créer de petites unités en subventionnant des abattoirs publics et salles de découpe. Les principaux industriels, au contraire, plaident en faveur de la concentration de l'activité, et, pour la favoriser, réclament que les subventions publiques soient réservées aux abattoirs privés d'entreprises justifiant d'un certain volume d'activité, ce qui reviendrait à une sélection spatiale en faveur de l'Ouest.

Ces conflits stratégiques (stratégies spatiales inconciliables) sont extrêmement fréquents dès que l'on s'intéresse à un ensemble aréal et non plus à l'analyse des stratégies individuelles. Il serait intéressant d'observer par exemple dans quelles conditions, au sein de la Rioja, s'insèrent les nouveaux groupes viticoles qui y multiplient les investissements.

21 Enfin, des stratégies qui, dans leurs finalités comme dans les discours qui les accompagnaient n'ont pas à l'origine de dimension spatiale, tels les quotas de production laitière en Europe à visée purement budgétaire, ont pu très vite générer un « effet territorial » important. D'une part, les aides à la création ont d'emblée posé la question du devenir des terres des exploitants âgés et de la valeur des herbages soustraits à la production laitière. D'autre part, la mesure conduit à toute une batterie de stratégies défensives au sein desquelles la localisation des acteurs, comme leurs formes de territorialité, ne sont pas neutres et produisent ensemble - et produiront car l'instabilité demeure - des dynamiques territoriales importantes qui mettent nettement en valeur les interactions fortes entre filières, produits et, au sens large, régions. 
$22 \mathrm{Au}$ fond, décider ou non qu'il y a stratégie spatiale ou territoriale dans les dynamiques observées revient sans doute à interpréter la nature et les délais de réaction des acteurs : peut-on dire qu'il y a "plan qui détermine leur choix dans chaque situation?" Et peut-on parler de stratégie lorsque les choix apparaissent prédéterminés, collectifs mais non explicites, telles, par exemple, les réactions à des applications de politiques sectorielles?

\section{NOTES}

1. Cf. dans ce numéro de STRATES : FAGNANI Jeanne, 1990. « De l'utilisation du concept de stratégie en géographie sociale : quelques éléments de réflexion ».

2. PINCHEMEL P. et G., 1989. La face de la terre, Armand Colin, Paris.

3. AURIAC F., 1983. Système économique et espace. Economica, Paris.

4. Cf. Dans ce numéro de STRATES : ROCHEFORT Michel et ROUSSEL Monique.

«Politique, stratégies et tactiques dans la dynamique urbaine : l'exemple de Brasilia ».

5. Cf. dans ce numéro de STRATES : LUGINBUHL Yves. «Plus stratégique que le territoire, tu meurs".

6. Cf. dans ce numéro de STRATES : GUERRINI Marie-Claude. « Systèmes de pouvoirs et gestion du territoire dans le sud du Parc national des Cévennes ».

7. Équipe «Analyse des espaces ruraux », 1983. Mécanismes et limites des processus de spécialisation/diversification de l'espace rural. Rapport de synthèse. DISPE 1, Laboratoire de Géographie Humaine/SEGESA, Paris, 126 pages.

\section{RÉSUMÉS}

La notion de "stratégie territoriale ", bien que très restrictive par rapport à l'ensemble du champ du concept de "stratégie ", s'avère fondamentale pour un certain nombre de recherches thématiques, par exemple sur les dynamiques foncières ou l'ancrage spatial des agro-industries. Elle apparaît en revanche moins immédiatement opératoire dans l'analyse des dynamiques spatiales complexes à propos desquelles son utilité est controversée. Sans forcément parler de "stratégies territoriales", une partie des chercheurs considère que l'intelligence des phénomènes ne saurait être acquise sans au moins la connaissance des stratégies d'acteurs multiples.

The notion of territorial strategies in STRATES rural and agro-industrial researches

The notion of «territorial strategy » which gives key-interpretation to rural and agro-industrial research works, appears to be less appropriate to the study of more complex research fields, the debate is widely opened and discussed by the anthor. 
INDEX

Mots-clés : espace rural, Politiques, Stratégie territoriale, action territoriale, Dynamique des espaces, Fonctionnement sociospatial, Local, Stratégie foncière, Systèmes de pouvoirs

Keywords : territorial strategy, Rural space, Power systems, Dynamics of spaces, Socio-spatial organization

\section{AUTEUR}

\section{FRANÇOISE PLET}

Maître de conférences à l'Université de Paris VIII, elle est spécialiste du domaine agroalimentaire. 